\title{
Effect of Originator Infliximab Treatment on Disease-Related Hospitalizations, Work Productivity and Activity Impairment, and Health Resource Utilization in Patients with Crohn's Disease in a Real-Life Setting: Results of a Prospective Multicenter Study in Germany
}

\author{
Niels Teich ${ }^{a}$ Michael Bläker ${ }^{b}$ Frank Holtkamp-Endemann ${ }^{c} \quad$ Eric Jörgensen ${ }^{d}$ \\ Andreas Stallmach ${ }^{\mathrm{e}}$ Susanne Hohenberger ${ }^{f}$ \\ anternistische Gemeinschaftspraxis für Verdauungs- und Stoffwechselkrankheiten Leipzig und Schkeuditz, \\ Leipzig, Germany; ${ }^{b}$ Gastroenterologie/Gastropraxis Eppendorfer Baum, Hamburg, Germany; 'Gastroenterologische \\ Gemeinschaftspraxis am Germania-Campus, Münster, Germany; ${ }^{d}$ Magen-Darm-Zentrum Remscheid, Remscheid,

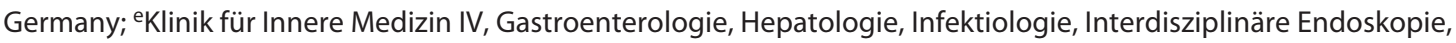 \\ Universitätsklinikum Jena, Jena, Germany; ${ }^{f}$ Medical Affairs, MSD Sharp \& Dohme GmbH, Haar, Germany
}

\section{Keywords}

Crohn's disease $\cdot$ Infliximab $\cdot$ Hospitalization rate $\cdot$ Work productivity/activity impairment $\cdot$ Health-related quality of life

\footnotetext{
Abstract

Introduction: Infliximab (IFX) therapy is efficacious for inducing and maintaining symptomatic remission in patients with Crohn's disease (CD), but whether this benefit results in reduced hospitalization rates and therefore may improve patients' quality of life in an economically sensible way is conflicting so far. Methods: We conducted a noninterventional, multicenter, open-label, prospective study to evaluate the effect of originator IFX treatment on patient-reported outcomes and disease-related hospitalizations in adult CD patients in Germany treated for the first time with IFX according to label. Results: Two hundred and ninety-four patients were included in the study. We observed a statistically significant reduction in the number of CD-related hos-
}

pitalizations from the year before baseline (mean 1.00 per patient, $S D \pm 0.93)$ to the mean value of the 1 st $(0.62, S D \pm$ $0.95)$ and 2 nd year $(0.32, S D \pm 0.75)$ of the observation period $(p<0.0001)$. After 3 months of IFX therapy, work productivity and activity increased by an average of 12.6 and $17.1 \%$, respectively. Patient's clinical outcome was markedly improved as the total $C D$ activity index (CDAl) sum score continuously decreased from baseline to month 24 and the mean score of the total inflammatory bowel disease questionnaire (IBDQ) changed substantially from 141 at baseline to 172 after 24 months of IFX treatment. Additionally, the number of work incapacity days declined. Recently, no new safety issues of IFX have been identified. Conclusion: In this large, prospective, multicenter study on disease-related hospitalization rates, work productivity, capacity for daily activities, and HRQoL in patients with CD, IFX significantly reduces their hospitalization rates and improves work productivity, daily activity, and quality of life over 24 months.

(c) 2020 The Author(s)

Published by S. Karger AG, Basel karger@karger.com www.karger.com/iid

Karger $\stackrel{\text { ' }}{5}$

BOPEN ACCESS
(C) 2020 The Author(s)

Published by S. Karger AG, Basel

This is an Open Access article licensed under the Creative Commons Attribution-NonCommercial-4.0 International License (CC BY-NC) (http://www.karger.com/Services/OpenAccessLicense), applicable to the online version of the article only. Usage and distribution for commercial purposes requires written permission.
Niels Teich

Verdauungs- und Stoffwechselkrankheiten

Internistische Gemeinschaftspraxis fürVerdauungs- und Stoffwechselkrankheiten

Nordstr. 21, DE-04105 Leipzig (Germany)

teich@igvs.de 


\section{Introduction}

Crohn's disease (CD) is a chronic inflammatory bowel disease (IBD) usually diagnosed in early adulthood. It is characterized by debilitating symptoms such as diarrhea, abdominal pain, anemia, and fatigue [1]. Persistent CD activity often causes reduced patients' work ability as well as patients' capacity to perform daily activities resulting in an impaired health-related quality of life (HRQoL) [2, $3]$. Furthermore, emergency hospital admissions are repeatedly necessary in a significant number of patients representing further disease burden $[2,4]$.

Infliximab (IFX), a monoclonal antibody against the anti-tumor necrosis factor alpha, is a proven-effective therapeutic agent in treatment of moderate to severe chronically active and fistulizing CD [5-7]. IFX and other anti-TNFs such as adalimumab offer significant benefits in $\mathrm{CD}$, but their continuous use leads to higher medication costs in the treatment of CD [8-10]. Nevertheless, disease-related hospitalizations and other socioeconomic effects of CD cause high utilization of health-care resources but might be minimized by an adequate treatment strategy [10-13]. So far, the effect of IFX on CDrelated hospitalizations and work ability impairment is inconsistently assessed [10,14, 15]. Usually, studies concerning CD-related hospitalizations focus on hospitalization costs, differences between older and younger $\mathrm{CD}$ patients, or compare different CD treatments and their influence on disease-related hospitalization rates $[10,14$, 15]. However, the extent to which more frequent hospitalizations lead to a worsening of HRQoL has mostly been neglected in previous studies. Van der Valk et al. [10] investigated the development of overall costs in patients suffering from IBD and demonstrated that costs remained stable over a time period of 2 years, although the proportion of anti-TNF-related health-care costs increased. The main reason was a reduction in hospitalization-related costs showing that an intensified use of anti-TNF therapy led to a decline in hospitalization rates but whether these findings were associated with changes in costs due to productivity loss or with an improvement of HRQoL was not determined by the authors. Another study, conducted by $\mathrm{Xu}$ et al. [15], evaluated the impact of IFX treatment of HRQoL in IBD patients in comparison to conventional therapies. Therefore, they analyzed the marriage status, employment, and economic burden in IBD patients in China. The study revealed that IFX treatment significantly improved the HRQoL and increased work time when compared with conventional therapies, but there was no assessment concerning a correlation between hospitaliza- tions and HRQoL. In our study, we aimed to overcome this lack of knowledge since both aspects are important factors in CD patients and evaluate whether a decline of hospitalization rates due to IFX treatment leads to improved work ability in CD patients.

The PRODUCTIVE (Improvement of Patient-Reported Outcomes during treatment with IFX in a real-life setting) study was designed to evaluate hospitalization rates and work impairment in CD patients in a real-life setting in Germany. Our aim was to provide useful information to enhance patients' quality of life (QoL) in a medically and economically sensible way.

\section{Materials and Methods}

\section{Study Population}

The PRODUCTIVE study was a noninterventional, multicenter, open-label, prospective study in Germany. Adult patients fulfilling the following inclusion criteria were enrolled: (1) a minimum age of 18 , (2) CD diagnosis by a gastroenterologist, (3) planned treatment with IFX in accordance to the respective summary of product characteristics (SmPC) [16] independently of including the patient into the study, and (4) given informed consent and agreement with documentation and evaluation of patient's data in a pseudonymized way. Patients with prior treatment with IFX (1), a switch to IFX from former biologic treatment because of serious adverse events (SAE), opportunistic infection, or allergic reaction (2), participation in another clinical trial (3), known hypersensitivity to the active compound, to other murine proteins, or to any of the excipients of the therapeutic drug (4), tuberculosis or other severe infections such as sepsis, abscesses, and opportunistic infections (5), and/or with moderate or severe heart failure (NYHA [New York Heart Association] class III/IV) (6) were excluded from the study. Women of childbearing age were intended to use adequate contraception to prevent pregnancy during IFX therapy as specified in the SmPC. Male patients and their female partners were also expected to use adequate contraception during and after IFX therapy. According to the SmPC, IFX was administered intravenously at a dose of $5 \mathrm{mg} / \mathrm{kg}$ body weight in weeks 0 , 2, and 6 mentioned as the induction phase (shown in Fig. 1a). Afterward, patients switched to maintenance therapy and received IFX every 8 weeks in the given dose via intravenous administration. Adjustment of dose and interval of administration was allowed as currently approved in CD.

\section{Data Collection}

Data were collected from 46 centers over 2 years starting with a baseline (BL) visit in month 0 followed by 6 post-BL visits in months $3,6,9,12,18$, and 24. Investigators had to document disease-related hospitalization, health-related work productivity/activity impairment (WPAI), and patients' QoL at all appointments. Disease-related hospitalization was assessed at BL and during observational period by physician's records, patient information, and discharge summaries from the hospital. The decision concerning relationship between hospitalization and CD was made upon physician's discretion. At BL, investigators also asked for disease-re- 


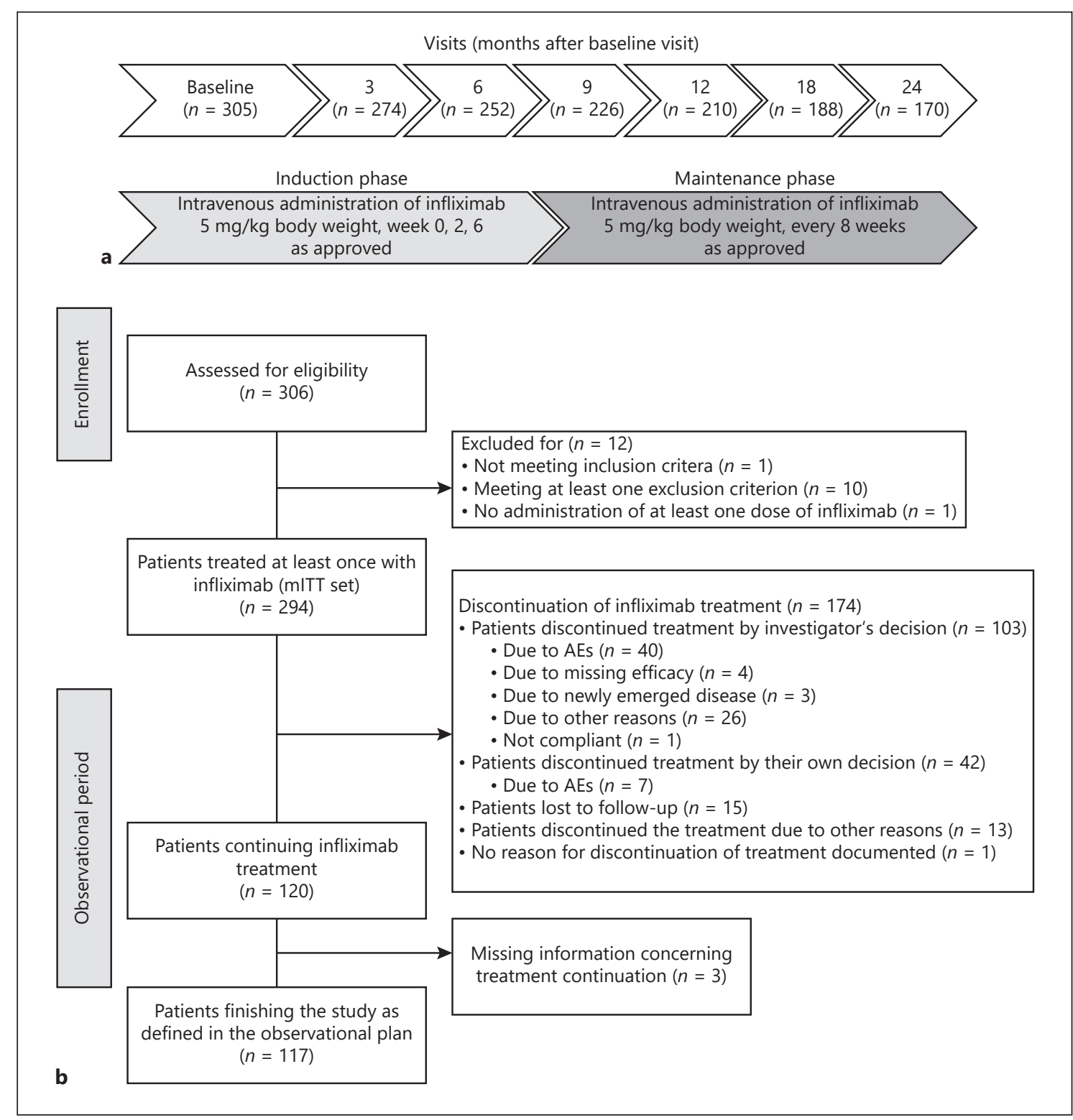

Fig. 1. Study design and patient flow diagram. a Overview of the study design. $\mathbf{b}$ Patient flow chart. $n$, number of patients; mITT, modified intention to treat; AE, adverse effect.

lated hospitalizations in the year prior to study initiation and for both the work incapacity days and the number of physician visits as well as non-physician visits during the 3 months before BL visit as initial situation previous to the start of IFX therapy. Reilly et al. [17] had demonstrated the discriminative validity, reliability, and responsiveness of the WPAI in 62 patients with CD in a prospective randomized 26-week clinical trial. Therefore, the WPAI questionnaire was used for evaluation of health-related WPAI, whereas for the overall health status, the ability to perform moderate activities, and patients' QoL, the Short Form 12 (SF-12) and the inflammatory bowel disease questionnaire (IBDQ) were used. Clinical outcomes during the observational period were assessed by Crohn's disease activity index (CDAI). A broad range of items in terms of health-care resource utilization (HCRU) such as number of physician visits, medication, physiotherapy, or psychotherapy were committed by the physician at all visits within the observational phase to enable the calculation of overall resource consumption. At each study visit, a complete physical examination was performed albeit vital signs including heart rate and blood pressure were just gathered at BL visit. All adverse events (AEs) during the observational phase were recorded including dates of onset and end, severity, relationship to IFX, any action taken, and outcome in accordance with ICH/GCP (International Conference on Harmonisation/good clinical practice) guidelines. 
Table 1. Patient demographics and vital signs at baseline

\begin{tabular}{|c|c|c|}
\hline Characteristic & Unit & Total $(n=294)$ \\
\hline Age, years & Median (range) & $33.5(18-74)$ \\
\hline \multicolumn{3}{|l|}{ Sex } \\
\hline Male & $n(\%)$ & $139(47.3)$ \\
\hline Female & $n(\%)$ & $155(52.7)$ \\
\hline \multicolumn{3}{|l|}{ BMI, $\mathrm{kg} / \mathrm{m}^{2}$} \\
\hline Male & Median (range) & $24(17.3-48.3)$ \\
\hline Female & Median (range) & $24(15.3-95.3)$ \\
\hline \multicolumn{3}{|l|}{ Systolic blood pressure, $\mathrm{mm} \mathrm{Hg}$} \\
\hline Male & Median (range) & $125(100-165)$ \\
\hline Female & Median (range) & $120(90-189)$ \\
\hline \multicolumn{3}{|l|}{ Diastolic blood pressure, $\mathrm{mm} \mathrm{Hg}$} \\
\hline Male & Median (range) & $78(55-110)$ \\
\hline Female & Median (range) & $80(45-118)$ \\
\hline \multicolumn{3}{|l|}{ Biologic-naïve } \\
\hline Yes & $n(\%)$ & $243(82.7)$ \\
\hline No & $n(\%)$ & $51(17.3)$ \\
\hline \multicolumn{3}{|l|}{ Nicotine abuse } \\
\hline Yes & $n(\%)$ & $93(31.6)$ \\
\hline Patients with tuberculosis & $n(\%)$ & $0(0)$ \\
\hline \multicolumn{3}{|l|}{ Patients with fistulae } \\
\hline Anal fistula & $n(\%)$ & $28(9.5)$ \\
\hline Rectovaginal fistula & $n(\%)$ & $1(0.3)$ \\
\hline Intra-abdominal and enterocutaneous fistula & $n(\%)$ & $6(2.0)$ \\
\hline Anal fissure & $n(\%)$ & $11(3.7)$ \\
\hline \multicolumn{3}{|l|}{ Concomitant medication } \\
\hline Azathioprine & $n(\%)$ & $91(31.0)$ \\
\hline Methotrexate & $n(\%)$ & $19(6.5)$ \\
\hline Cortisone & $n(\%)$ & $17(5.8)$ \\
\hline Prednisolone & $n(\%)$ & $18(43.5)$ \\
\hline
\end{tabular}

\section{Outcome Measurements}

The primary end point was the absolute change in diseaserelated hospitalizations per year between the computed previous number of hospitalizations per patient and year before BL and mean value of the observed number of hospitalizations per patient per 1st and 2 nd year of the observational period. Percentage change in total work productivity impairment (TWPI) and activity impairment using the WPAI between BL and month 3 represented the co-primary end point. Secondary objectives were to assess the changes in disease-related hospitalizations per year between the historical (previous) number of hospitalizations per patient and year and the observed number of hospitalizations per patient separately for the 1 st and the 2 nd year of the observation period as well as the percentage change in the TWPI and activity impairment and the percentage change in work time missed (absenteeism) and impairment at work (presenteeism), all assessed using the WPAI between BL and months 3, 6, 9, 12, 18 , and 24. The absolute change in SF-12 between BL and month 3 and the absolute change in IBDQ and in CDAI between BL and months $3,6,9,12,18$, and 24 represented further secondary objectives. Additionally, the change in disease-related HCRU between 3 months before $\mathrm{BL}$ and during the observational phase was analyzed. All AEs and SAEs during the observational period were documented for an enlarged safety analysis of IFX treatment.

\section{Statistical Analysis}

Statistical analyses were performed using SAS version 9.4. Categorical variables are presented as numbers and percentages. Quantitative variables are shown as mean with SD or median with range. Differences or changes from $\mathrm{BL}$ are indicated as mean change with SD or percentage change (\%). All enrolled patients who received at least one dose of IFX were included in the modified intention-to-treat (mITT) analysis set and considered for evaluation if data were available for analysis of the respective end point. Data from visits assessed during the observational phase but after early discontinuation of treatment or during treatment break were kept in the mITT analysis set. For those patients who early discontinued the study prior to visit month 24 , the number of disease-related hospitalizations per year was extrapolated (either for the 1st or 2nd year of observation period) as (number of hospitalizations $\times 365$ [days])/observation period (days), with observation period $=$ date of last visit (end) - date of BL (month 0 ). For the main end point parameter, a subgroup analysis of biolog- 
ical-naïve versus biological pre-treated patients was performed. Demographic data (age, sex, marital status, occupational status, and pre-treated with biologics or not), BL vitality data (body height, body weight, BMI, blood pressure, and heart rate), ensured contraception, any substance abuse, tuberculosis anamnesis, as well as previous and concomitant medications were summarized descriptively. Disease-related HCRU was also descriptively assessed for physician visits, non-physician visits, rehabilitation, incapacity to work, and level of care. The primary, the co-primary, and the secondary end point variables were calculated with summary statistics. WPAI results were expressed as impairment percentages, with higher numbers indicating greater impairment and less productivity. WPAI outcomes (with exception of question Q6 and the activity impairment) were evaluated and analyzed only for patients who were employed during the study periods (before BL, 1 st year, 2 nd year, and mean of 1 st and 2 nd year). A repeated measurement analysis was conducted on WPAI, SF-12, IBDQ, and CDAI data to identify any changes on outcomes during the observational period (visits). The SF-12 domains were calculated by the software Optum PRO CoRE version 1.3. For each IBDQ domain, a total score and average per item scores were calculated and analyzed. A paired $t$ test was conducted to analyze changes in primary, co-primary, and secondary end points. If case data were nonnormally distributed, the Wilcoxon signed-rank test was to be used.

\section{Results}

\section{Patient Demographics and Therapy Details}

From December 6, 2012, to September 25, 2018, 306 patients were screened at 46 sites in Germany for participating in the PRODUCTIVE study. Finally, 294 eligible patients from 46 sites provided written informed consent and were included in the study (mITT analysis set, shown in Fig. 1b). In total, 170 patients completed the study after last visit in month 24 . The gender-balanced study population (female $52.7 \%$ ) had a median age of 34 years ( $\min 18$, max 74 years, shown in Table 1). At BL, the majority of patients were full-time employed (50.0\%) or a study/trainee (12.2\%). Part-time employment was more often due to other reasons $(8.5 \%)$ than to CD (4.4\%). The majority of patients $(82.7 \%)$ were biological-naïve before initiating the study. Most patients required concomitant IBD medication (91.2\%), from which prednisolone and azathioprine were the most commonly taken concomitant drugs by 43.5 and $31.0 \%$ of the patients, respectively. Perianal fistulae were reported in $9.5 \%$ of the patients. The median overall treatment period with originator IFX was 66 weeks (range: 0-131 week, $n=291$ patients), and the mean duration of observation was 2 years (range: $0-3$ years). In sum, $59.2 \%$ of all the patients terminated IFX therapy prematurely (shown in Fig. 1b). In most of the patients $(n=$
103), treatment was stopped by investigator's decision due to AEs $(n=40)$, missing efficacy $(n=34)$, or other reasons $(n=29)$ such as remission, surgical interventions, or switch to a biosimilar, whereas 42 patients terminated treatment by their own decision. Finally, 117 patients received originator IFX therapy for the entire observation period of 24 months.

\section{Disease-Related and Non-Disease-Related}

Hospitalization in IFX-Treated Patients with CD

The mean number of hospitalizations per year decreased continuously from 1.00 in the year before observation to 0.62 in the 1 st year and to 0.32 in the 2 nd year of IFX therapy (statistically significant change in the Wilcoxon signed-rank test with $p=0.0005$ [ 1 st year] and $p<0.0001$ [2nd year], respectively, shown in Fig. 2a). The mean number of hospitalizations during the 2-year observational phase (sum of 1 st and 2 nd year) of IFX therapy was 0.55 and dropped markedly by 0.45 compared to the number in the year before the study was initiated (statistically significant in the Wilcoxon signedrank Test, $p<0.0001)$. Subgroup analyses of the primary endpoint revealed statistically significant changes in disease-related hospitalizations between the year before observation and the 1 st year (mean difference $0.51, p<$ 0.0001 , Wilcoxon signed-rank test), the 2 nd year (mean difference $0.80, p<0.0001$, Wilcoxon signed-rank test), and the whole 2 years (mean difference $0.60, p<0.0001$, in both Wilcoxon signed-rank test and paired $t$ test) of IFX therapy in biological-naïve patients versus patients pre-treated with biologics (shown in Fig. 2c). The number of non-disease or therapy-related hospitalizations per year was evaluated for only about 20 patients. Our analysis exhibited that the mean number of non-disease or therapy-related hospitalization decreased continuously and substantially from 0.73 in the year before BL to 0.62 in the 1 st year and to 0.21 in the 2 nd year of observation (shown in Fig. 2b). We further had a closer look on the number of days in hospital and detected an obvious and sustained decrease in the mean duration of hospitalizations per year with a mean decline of the average duration of 2.1 days ( $p \leq 0.0010$, Wilcoxon signedrank test) in the 1 st year and 4.2 days $(p<0.0001$, Wilcoxon signed-rank test) in the 2 nd year of IFX therapy (shown in Fig. 2d). Regarding the entire 2-year observational period, the average duration of hospitalizations was 2.5 days and dropped by 3.7 days compared to the duration in the year before the observation $(p<0.0001$, Wilcoxon signed-rank test).
Teich/Bläker/Holtkamp-Endemann/ Jörgensen/Stallmach/Hohenberger 


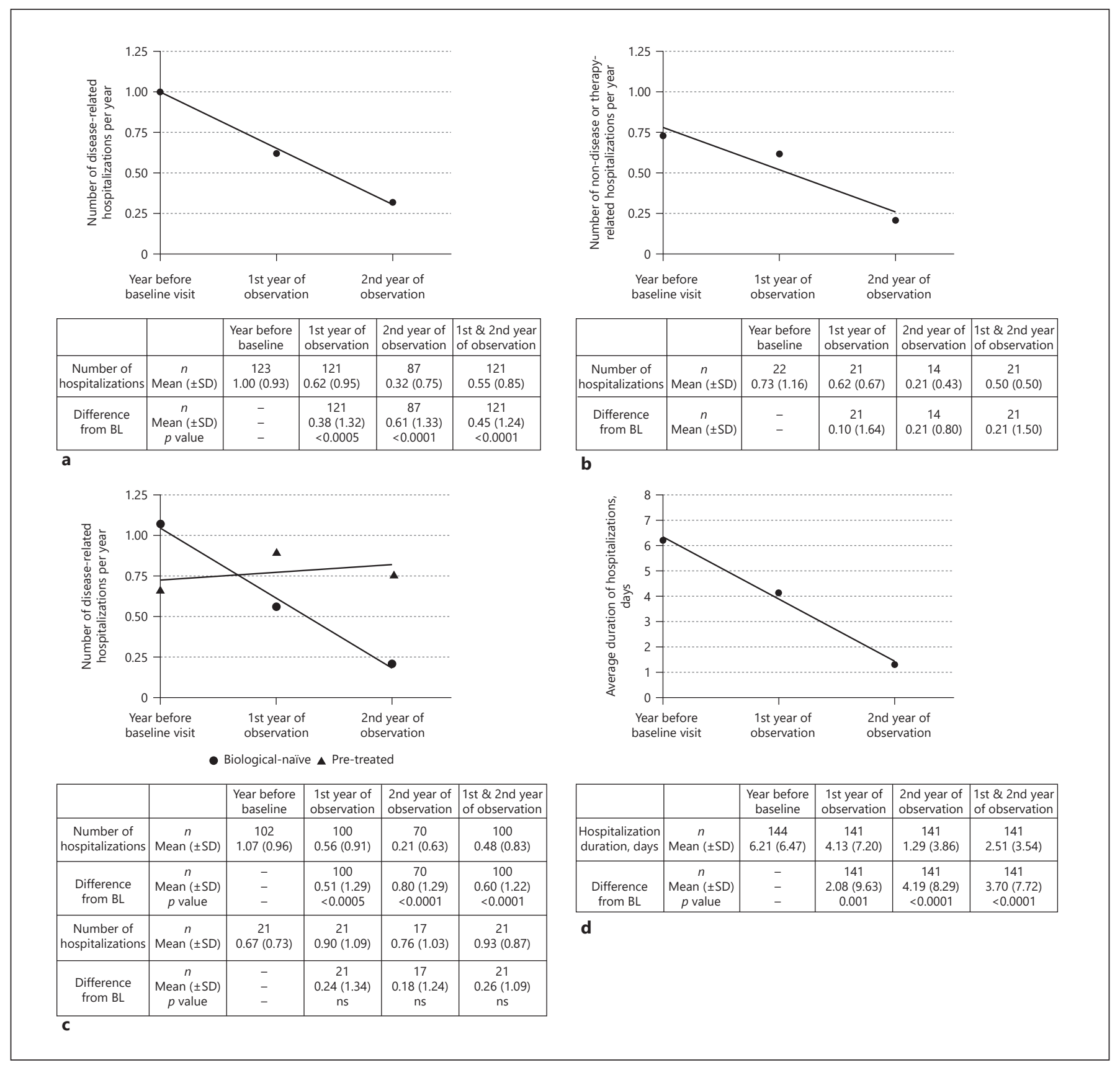

Fig. 2. Disease-related and non-disease-related hospitalizations in CD patients. The number of disease-related hospitalizations is shown for all patients (a) and for biological-naïve ( $)$ patients in comparison to pre-treated $(\mathbf{\Delta})$ patients $(\mathbf{c})$ for the year before BL visit and for the 1st and 2 nd year of observation. Before BL means the number of diseaserelated hospitalizations documented during the 12 months before $\mathrm{BL}$ visit until BL visit (month 0). First year of observation represents the overall number of hospitalizations during the 1st year of observation which is counted from the day of BL visit +1 until visit month 12 . The 2nd year of observation sums up the number of hospitalizations during the 2 nd year with an observation period starting on the day of visit in month $12+1$ until visit month 24 . For patients who early discontinued the study prior to visit month 24 , the number of disease-

related hospitalizations per year was extrapolated (either for the 1st or 2nd year of the observation period) as (number of disease-related hospitalizations $\times 365$ [days])/observation period (days), with observation period $=$ date of last visit (end) - date of $\mathrm{BL}$ (month 0 ). b Displays the non-disease or therapy-related number of hospitalizations showing no statistically significant results. The average duration of hospitalization is shown in (d). Statistical analyses were done on the descriptive model, and $p$ values were calculated by the Wilcoxon signed-rank test for the mean difference from BL for the 1st, the 2nd, and the mean of the 1 st and 2 nd year. Linear regression lines were calculated using GraphPad Prism 7.05. ns, not significant; $n$, number of patients with available data for the respective time point; $C D$, Crohn's disease; BL, baseline. 


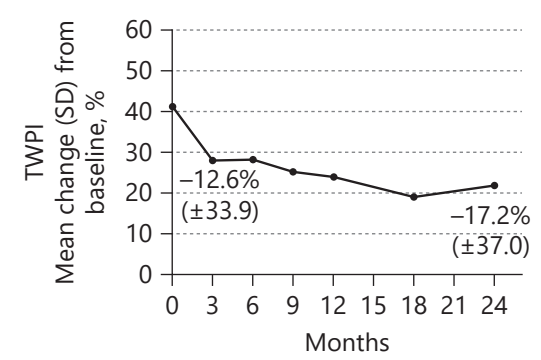

\begin{tabular}{|c|c|c|c|c|c|c|c|}
\hline & & $\begin{array}{c}\text { Month } \\
3\end{array}$ & $\begin{array}{c}\text { Month } \\
6\end{array}$ & $\begin{array}{c}\text { Month } \\
9\end{array}$ & $\begin{array}{c}\text { Month } \\
12\end{array}$ & $\begin{array}{c}\text { Month } \\
18\end{array}$ & $\begin{array}{c}\text { Month } \\
24\end{array}$ \\
\hline \multirow{2}{*}{$\begin{array}{c}\text { Change } \\
\text { from }\end{array}$} & $n$ & 91 & 90 & 79 & 75 & 73 & 66 \\
BL & $\begin{array}{c}\text { Mean } \\
( \pm S D)\end{array}$ & -12.6 & -14.3 & -16.9 & -18.5 & -20.7 & -17.2 \\
& $p$ value & 0.0003 & $<0.0001$ & $<0.0001$ & $<0.0001$ & $<0.0001$ & 0.0003 \\
\hline
\end{tabular}

a
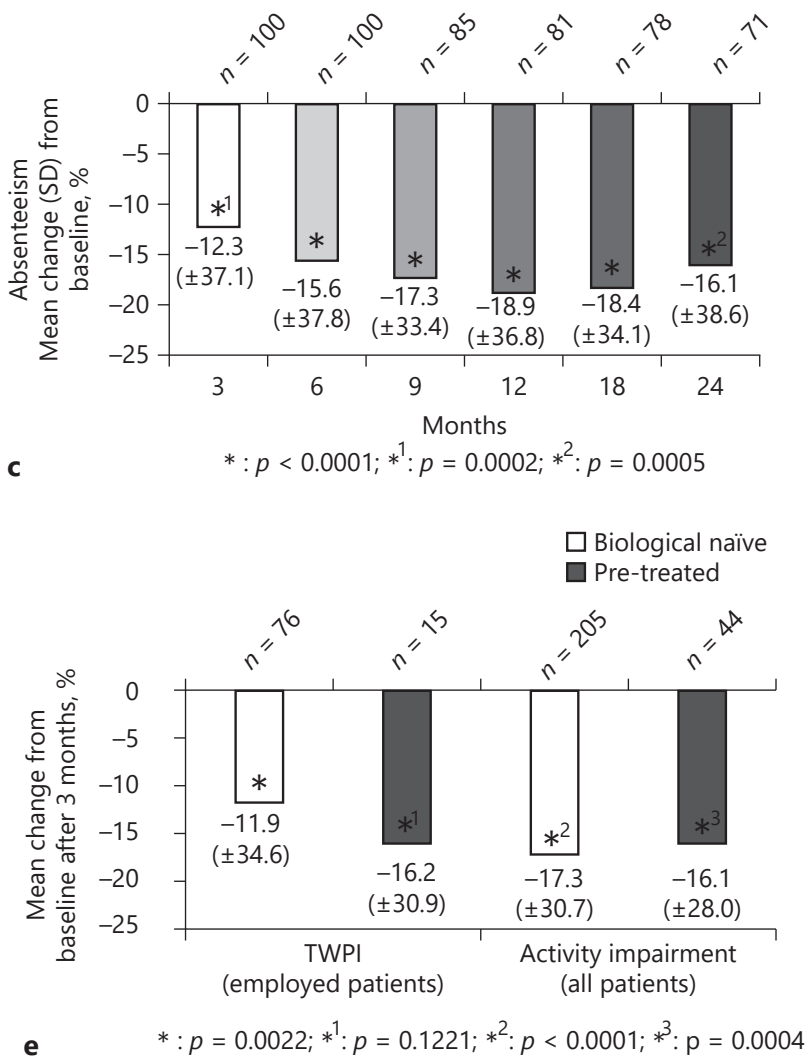

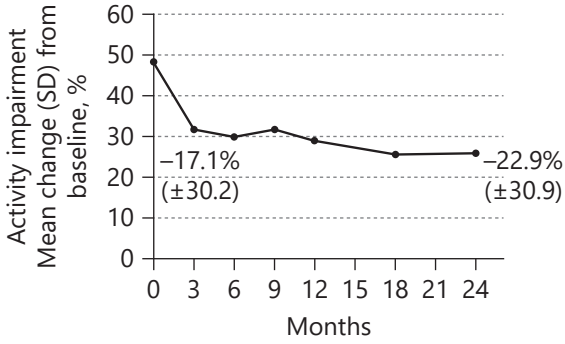

\begin{tabular}{|c|c|c|c|c|c|c|c|}
\hline & & $\begin{array}{c}\text { Month } \\
3\end{array}$ & $\begin{array}{c}\text { Month } \\
6\end{array}$ & $\begin{array}{c}\text { Month } \\
9\end{array}$ & $\begin{array}{c}\text { Month } \\
12\end{array}$ & $\begin{array}{c}\text { Month } \\
18\end{array}$ & $\begin{array}{c}\text { Month } \\
24\end{array}$ \\
\hline \multirow{2}{*}{ Change } & $n$ & 249 & 227 & 207 & 194 & 173 & 154 \\
from & Mean & -17.1 & -17.2 & -16.4 & -19.2 & -23.0 & -22.9 \\
BL & $( \pm S D)$ & $(30.2)$ & $(33.1)$ & $(31.0)$ & $(31.9)$ & $(32.9)$ & $(30.9)$ \\
& $p$ value & $<0.0001$ & $<0.0001$ & $<0.0001$ & $<0.0001$ & $<0.0001$ & $<0.0001$ \\
\hline
\end{tabular}

b

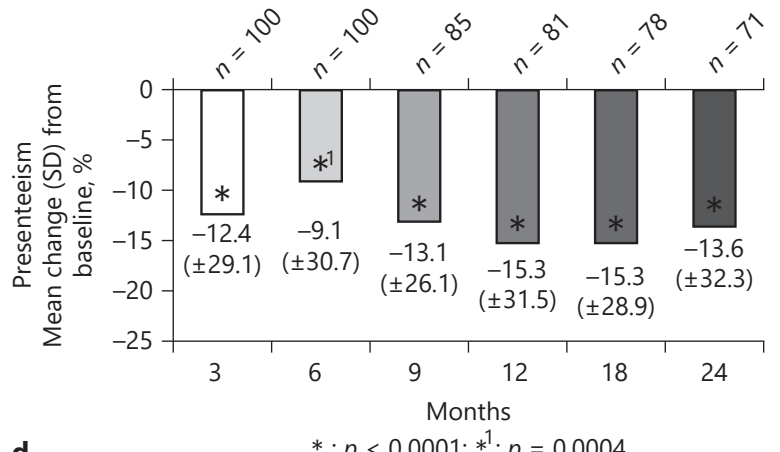

$*: p<0.0001 ; *^{1}: p=0.0004$

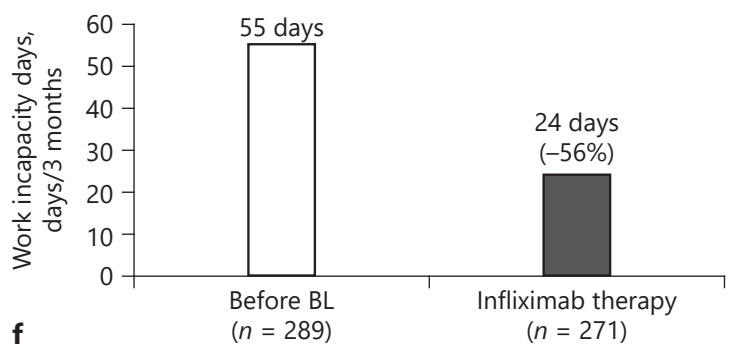

Fig. 3. Work productivity and activity impairment in CD patients. TWPI (a) and activity impairment (b) are shown for the whole observational period. After the onset of IFX therapy, both impairments were statistically significantly improved. For absenteeism (c) as well as presenteeism (d), similar outcomes were reported. e Comparison of TWPI and activity impairment between biological-naïve (white columns) and pre-treated (dark gray columns) patients demonstrating a statistically significant enhancement in both groups for TWPI and activity impairment, respectively, after 3 months of IFX treatment (mean change from BL). The decrease of work incapacity days before BL and after 3 months of IFX therapy is shown in (f) indicated as days/3 months. a-e Analyses were performed in the mITT analysis set using the descriptive model. $p$ values were generated by the Wilcoxon signed-rank test and are pointed under the respective graphs. Data are shown as percentage mean with SD. $n$ number of patients with available data for the respective time point; CD, Crohn's disease; TWPI, total work productivity impairment; IFX, infliximab; BL, baseline; mITT, modified intention to treat. 
Fig. 4. QoL and PROs. The absolute change in IBDQ (a) and CDAI (c) is shown as mean with SD and was statistically significant for all visits (months 3-24) compared to $\mathrm{BL}$ (month 0 ). The mean change for each of the 4 domains of the IBDQ is presented in (b) revealing statistically significant changes for all items in months 3 and 24 in comparison to BL. Analyses were done for the mITT analysis set, and $p$ values were generated using the Wilcoxon signed-rank test $(*)$ or paired $t$ test $\left({ }^{* 1}\right)$. $n$, number of patients with available data for the respective time point. QoL, quality of life; PRO, patient-reported outcome; IBDQ, inflammatory bowel disease questionnaire; CDAI, Crohn's disease activity index; BL, baseline; mITT, modified intention to treat.

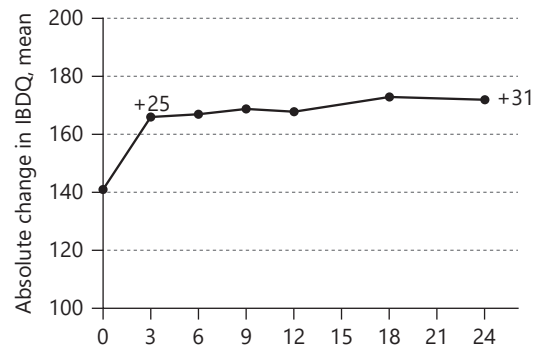

\begin{tabular}{|c|c|c|c|c|c|c|c|c|}
\hline & & BL & Month 3 & Month 6 & Month 9 & Month 12 & Month 18 & Month 24 \\
\hline Absolute & $n$ & 283 & 250 & 224 & 206 & 194 & 172 & 152 \\
score & Mean $( \pm$ SD) & $141(36)$ & $166(34)$ & $167(36)$ & $169(36)$ & $168(35)$ & $173(34)$ & $172(36)$ \\
\hline Change & $n$ & - & 243 & 219 & 201 & 189 & 168 & 147 \\
from & Mean $( \pm$ SD) & - & $25(32)$ & $24(35)$ & $26(35)$ & $26(36)$ & $33(35)$ & $31(36)$ \\
BL & $p$ value & - & $<0.0001^{b}$ & $<0.0001$ & $<0.0001^{b}$ & $<0.0001$ & $<0.0001$ & $<0.0001$ \\
\hline
\end{tabular}

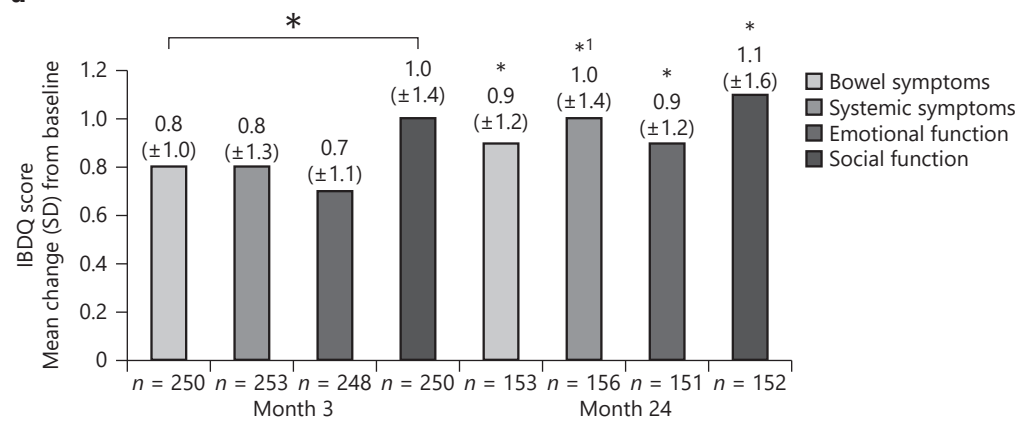

b $\quad *: p<0.0001$ (Wilcoxon signed-rank test); ${ }^{* 1}: p<0.0001$ (paired $t$ test)

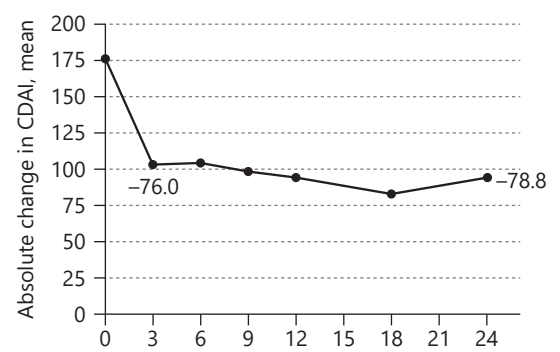

\begin{tabular}{|c|c|c|c|c|c|c|c|c|}
\hline & & BL & Month 3 & Month 6 & Month 9 & Month 12 & Month 18 & Month 24 \\
\hline \multirow{2}{*}{ Absolute } & $n$ & 255 & 229 & 207 & 195 & 180 & 156 & 143 \\
score & Mean & 186.8 & 103.7 & 105.0 & 98.8 & 95.0 & 83.2 & 94.5 \\
& $( \pm$ SD) & $(124.1)$ & $(94.7)$ & $(105.3)$ & $(104.4)$ & $(87.9)$ & $(86.6)$ & $(92.8)$ \\
\hline \multirow{2}{*}{ Change } & $n$ & - & 215 & 190 & 176 & 164 & 142 & 130 \\
from & Mean & - & -76.0 & -72.3 & -80.0 & -77.5 & -97.0 & -78.8 \\
BL & $( \pm$ SD $)$ & - & $(109.8)$ & $(127.9)$ & $(124.2)$ & $(127.0)$ & $(137.2)$ & $(123.5)$ \\
& $p$ value & - & $<0.0001$ & $<0.0001$ & $<0.0001$ & $<0.0001$ & $<0.0001$ & $<0.0001$ \\
\hline
\end{tabular}

\section{Changes in Work Productivity and Activity Impairment}

At BL, patients showed a mean TWPI of $41.2 \%(n=$ 144 patients $)$ and activity impairment of $48.6 \%(n=288$ patients), respectively (shown in Fig. 3a, b). During IFX therapy, the work productivity impairment as well as the activity impairment decreased continuously. TWPI dropped to $28 \%$ in months 3 and 6 and to $22 \%$ in month 24 . Activity impairment also dropped notably to approximately $30 \%$ at months $3,6,9$, and 12 and to $26 \%$ at months 18 and 24. In summary, we observed a statistically significant change from BL of $-12.6 \%$ in TWPI $(n=$ 91, $p=0.0003$, Wilcoxon signed-rank test) and $-17.1 \%$ ( $n=249, p<0.0001$, Wilcoxon signed-rank test) in activ- 
Fig. 5. Disease-related HCRU. Disease-related HCRU were assessed by the number of physician (black bars) and non-physician (blank bars) visits over the 3-month period as indicated. Alternative services or out-patient visits were counted among non-physician visits. HCRUs documented with start of utilization at most 90 days prior to BL visit (month 0 ) were defined as "before BL." For post-BL periods, the start date of any HCRU is allocated to the respective visit period. The numbers of HCRUs with date 90 days prior to the date of the respective visit are used, regardless of the exact duration between the visits and the start date of the HCRU. $n$, number of patients with available data for the respective time point; nd, no visits were documented (number of visits $=0$ ); HCRU, health-care resource utilization; BL, baseline.

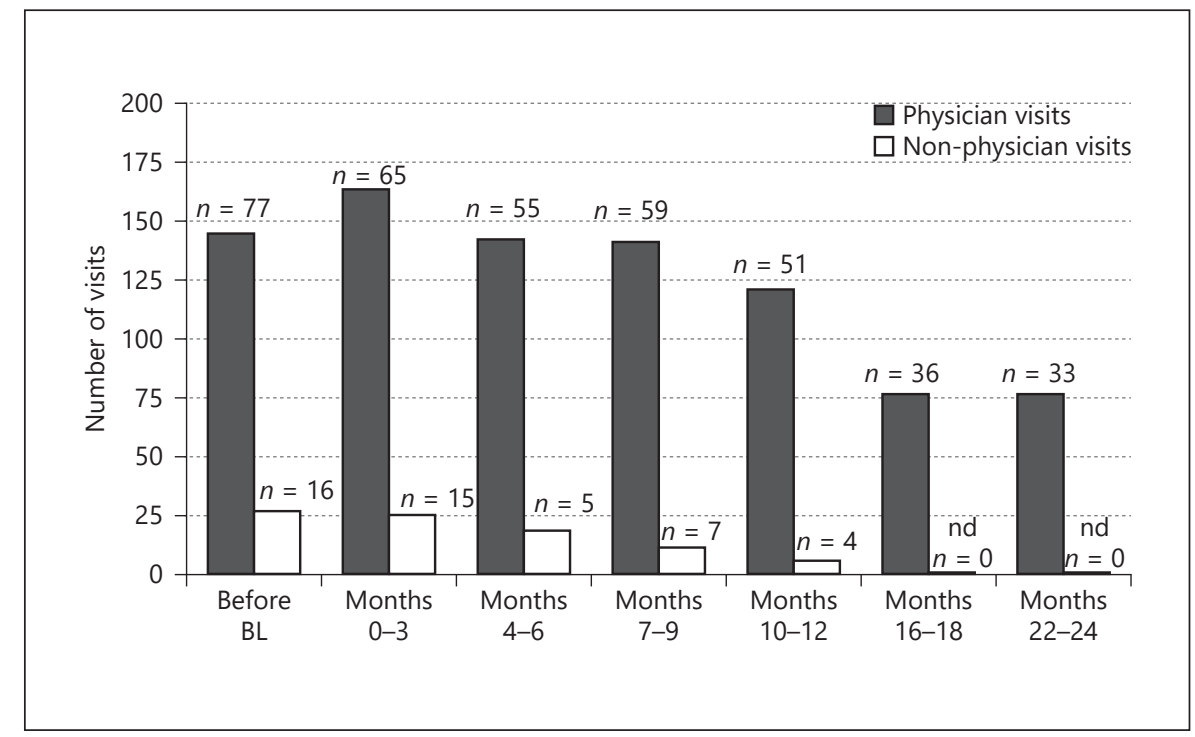

ity impairment after 3 months of IFX therapy. Having a closer look at the defined subgroups, TWPI decreased statistically significantly from BL visit to month 3 of the IFX therapy in biologic-naïve patients $(-11.9 \%, p=0.0022$ and $-17.3 \%, p<0.0001$, both in the Wilcoxon signedrank test) and in patients pre-treated with biologics $(-16.2 \%, p=0.1221$, Wilcoxon signed-rank test and $-16.1 \%, p=0.0004$, paired $t$ test, shown in Fig. 3e). For absenteeism as well as presenteeism, we also detected a statistically significant decline during the IFX therapy (shown in Fig. 3c, d). The mean work time missed decreased by $>10 \%$ from BL ranging from -12.3 (month 3 ) to $-18.9 \%$ (month 12 , for all changes $p \leq 0.0005$, Wilcoxon signed-rank test). A mean change from -12.4 (month 3) to $-15.3 \%$ (months 12 and 18 , for all changes $p \leq$ 0.0004 , Wilcoxon signed-rank test) was assessed for presenteeism during the observational phase. Additionally, the work incapacity days decreased from 55 days during the 3 months before BL to 24 days/ 3 months during IFX therapy (shown in Fig. 3f).

\section{QoL and Patient-Reported Outcomes}

HRQoL was assessed using the SF-12 and the IBDQ. Our data revealed that the total and mental health component score as well as all individual norm-based subscores assessed by the SF- 12 were improved after 3 months of IFX therapy with mean changes ranging from 3.4 to 7.0 (for all changes $p<0.0001$, Wilcoxon signed-rank test or paired $t$ test, shown in online suppl. Fig. 1; for all online suppl. material, see www.karger.com/doi/10.1159/000512159). This enhancement of HRQoL had been observable over the entire time of IFX therapy. Evaluation of the IBDQ also revealed an improvement of HRQoL as the mean score of the total IBDQ (covering bowl symptoms, systemic symptoms, emotional function, and social function, items added up) had changed from 141 at BL to 166 scores at month 3 and to 172 scores after 24 months with statistically significant changes from BL to each visit $(p<0.0001$, Wilcoxon signed-rank test or paired $t$ test, shown in Fig. 4a). Having a closer look at the mean change of the 4 IBDQ domains, an improved HRQoL was statistically significant for all items over the whole observational time $(p<0.0001$, Wilcoxon signed-rank test or paired $t$ test, shown in Fig. 4b). Patients' clinical outcome, reported by the CDAI score, was markedly improved clearly recognizable by a decrease of the total CDAI sum score from 177 at BL to 95 at month 24 (for all changes $p<0.0001$, Wilcoxon signed-rank test, shown in Fig. 4c).

\section{Health-Care Resource Utilization}

Besides the disease-related burdens in CD patients, we further evaluated the CD-related HCRU by analyzing the number of physician and non-physician visits within the 3 -month period in comparison to the 3 months prior to study initiation. In the first 3 months of IFX therapy, the number of physician visits (163) increased, whereas the number of non-physician visits (25) was approximately unchanged compared to the 3 months before BL ( 145 and 27, respectively, shown in Fig. 5). At the end of the observational phase, the number of visits at both, physician visits and non-physician visits, was considerably lower with 76 and 0 for months $16-18$ and $22-24$, respectively. 


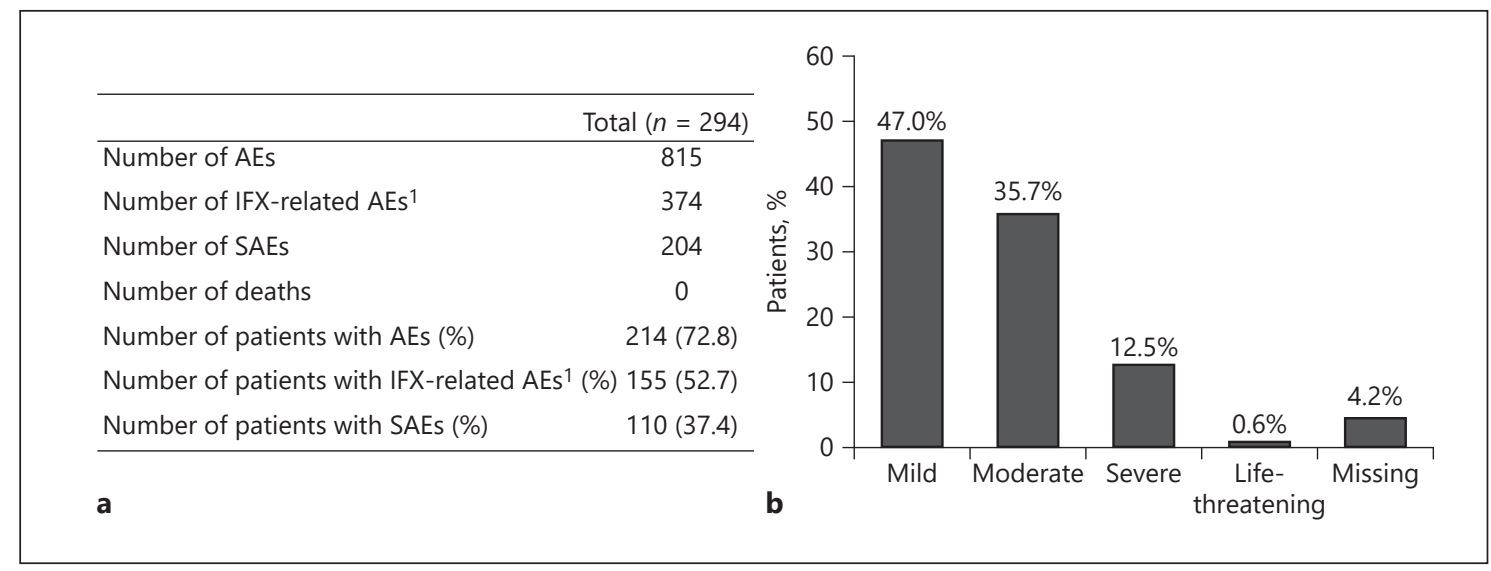

Fig. 6. Safety report. An overview of all AEs is given in (a), whereas in (b), the intensity of all AEs in the patient population is shown. Percentages are based on the total number of patients $(n=294) .{ }^{1}$ AEs were considered to be related to IFX or not judged. AEs, adverse events; IFX, infliximab; SAEs, serious adverse events.

For rehabilitation or convalescence treatment, no changes had been observable during the observational period.

\section{Safety Report}

In total, 815 AEs were documented during the observational phase (shown in Fig. 6a). For $73 \%$ of the patients (214 of 294), at least $1 \mathrm{AE}$ was reported. Forty-six percent of the AEs (374 of 815) were considered to be related to the IFX treatment by the respective physician, and at least $53 \%$ of all patients $(n=156)$ had been affected by an IFXrelated $\mathrm{AE}$. The most commonly reported system organ classes (SOCs) concerning AEs were gastrointestinal disorders and infections and infestations which occurred in $32 \%(n=95)$ and $29 \%(n=85)$ of the patients, respectively. Within the SOCs, most of the patients were affected by diarrhea $(8 \%, n=24)$ and nasopharyngitis $(10 \%$, $n=29)$. Interestingly, in $12 \%$ of the patients $(n=34)$, the $\mathrm{AE}$ "drug ineffective" was reported belonging to the SOC general disorders and administration site conditions. Most of the AEs were documented as mild or moderate, but 102 AEs were classified as severe (shown in Fig. 6b). One-third $(n=37)$ of severe AEs was considered as related to study medication (shown in Fig. 6a). SAEs were documented in $37.4 \%$ of the patients $(n=110)$ followed by therapy discontinuation in most of the cases. In 4 patients, a total of 5 life-threatening SAEs were reported. The remaining SAEs were mainly documented as mild, moderate, or with no specified intensity (total $n=124$ ), whereas 75 SAEs were notified as severe. A total of 51 SAEs were considered to be IFX related. Hospitalization was required by 11 of these SAEs while additional medi- cation was needed in 21 and another therapy was indicated in 14 SAEs. Finally, most of the SAEs and IFX-related SAEs had resolved or resolved with sequelae, and no death occurred during the observational period.

\section{Discussion/Conclusion}

In this large prospective multicenter study on diseaserelated hospitalization rates, work productivity, capacity for daily activities, and HRQoL in patients with CD, IFX significantly reduces their hospitalization rates and improves work productivity, daily activity, and QoL in CD patients over 24 months.

Our finding of a marked reduction in the number of hospitalizations per year in the 1st and 2nd year of IFX therapy demonstrated that originator IFX attenuated effectively symptoms of $\mathrm{CD}$, and therefore, patients starting IFX could be constrained to a less disease-related hospitalization. Furthermore, the PRODUCTIVE study revealed a considerably reduced duration of hospitalization which supports previous studies showing both a reduction in the mean number of hospitalizations as well as less hospitalization days in patients who received IFX either in comparison to patients obtaining placebo maintenance [18] or to patients without adherence to IFX maintenance therapy $[19,20]$. A decreased risk of hospitalization was also shown for adalimumab used in the CHARM study [21]. In addition to that, a Canadian study analyzing retrospectively patients treated with IFX showed a significant decrease in hospitalization once IFX was added [22]. 
In this study, patients served as their own control. In the PRODUCTIVE study, we chose the same approach in order to determine the change in hospitalization due to the applied treatment and to receive an assessment of HRQoL for a certain patient since disease-related hospitalizations should be considered as a subjectively perceived disease burden in CD patients. However, using such an approach also has a potential kind of bias since information given by patients is non-standardized and might be incomplete.

Besides the number of disease-related hospitalizations, the number of non-disease-related hospitalizations also declined. Nevertheless, in our study, the reasons for non-disease-related hospitalizations were not ascertained and the number of patients with available data for this endpoint was considerably low. The physician decided without predefined criteria whether the hospitalization was CD-related or not. These weaknesses do not allow a conclusion regarding cause and severity of non-CD-related hospitalizations.

The PRODUCTIVE study confirmed patients' improvement in HRQoL as evaluation of the IBDQ revealed an increase in IBDQ score after 3 months of IFX therapy which persisted over the whole observational period. Subsidiarily, the total and mental health component score assessed by the SF-12 was also enhanced after the onset as well as during IFX therapy. Finally, a significant improvement of the CDAI score was observed.

Several publications postulated a reduction in hospitalization and surgical interventions due to improved CD course in patients who respond to biologic-based treatment $[4,23]$. A systematic review with meta-analysis confirmed these studies as they noted statistically significant reductions in yearly hospitalizations in this recent random effects meta-analysis for both IFX (OR 0.45, 95\% CI 0.32-0.63) and - less-intensive - adalimumab (OR 0.50, 95\% CI 0.32-0.79). In addition to that, Mao et al. [24] demonstrated superiority of IFX and adalimumab to azathioprine in preventing CD-related hospitalization. Further restrictions in CD patients' QoL are often caused by work-life impairment [25]. Results from studies analyzing the work productivity loss in other gastrointestinal diseases suggest that $\mathrm{CD}$ patients are challenged with disease-specific embarrassments during working time [26, 27] such as abdominal bloating, diarrhea, and fatigue. Using the validated WPAI tool [17], we were first able to show that absenteeism and presenteeism decreased substantially during maintenance with originator IFX. Furthermore, statistically significant declines in TWPI and activity impairment with a pronounced effect in biological-naive patients within the first 3 months of IFX treat- ment in comparison to biological pre-treated patients were observed. As mentioned before, additionally used patient-reported outcomes (IBDQ and SF-12) demonstrated that IFX-induced improvement of HRQoL was obviously detectable in the study population revealing that originator IFX seems to enable CD patients a "normal" work life with less absenteeism and presenteeism rates and a lower number of work incapacity days.

Circumstances negatively affecting working time might cause psychical problems and self-doubts. This mostly hampers young adult patients who are at the beginning of their carrier when they have to make their pioneering career steps [28]. The PRODUCTIVE study is one initial study assessing TWPI in patients before the onset of IFX treatment and after 2 years of IFX therapy combined with patient-reported outcomes to evaluate HRQoL. We demonstrated that IFX treatment successfully restored patients' work life as well as HRQoL due to an improvement of disease symptoms. The same outcome was already shown for certolizumab [29] and adalimumab [30].

Besides the patients themselves, $\mathrm{CD}$ also compromises the society due to increasing total costs of this chronic disease [31-33]. CD is associated with unemployment, sick leaves, and claiming of disability pensions leading to high utilization of health services $[9,34,35]$. Additionally, high presenteeism rates in CD patients cause economic impairment of employer and further increase the indirect costs of the disease [36]. Our study demonstrated a decline in TWPI and a reduced number of hospitalizations after the onset of IFX treatment. These results persisted over the whole observational period of 2 years revealing a sustained effect of IFX maintenance in CD patients and therefore may contribute to a reduction of disease-related costs.

Finally, our study revealed no new safety issues on originator IFX, which is in line with other recent reports [37]. In summary, the PRODUCTIVE study reinforced that the usage of the originator IFX constituted an effective and well-tolerated therapy in $\mathrm{CD}[6,25]$ resulting in reduced hospitalization rates which in turn had a positive effect on work life and HRQoL.

\section{Acknowledgements}

The authors would like to thank the contract research organization FGK Clinical Research GmbH, Munich, Germany (funded by MSD SHARP \& DOHME GmbH, Haar, Germany) that was responsible for data management, statistical analysis, and report writing. For medical writing assistance in drafting and revision of the manuscript, we would like to thank Dr. Katharina Bakhaus, Alcedis $\mathrm{GmbH}$, Giessen, Germany, funded by MSD SHARP \& DOHME GmbH, Haar, Germany.
58

Inflamm Intest Dis 2021;6:48-60 DOI: $10.1159 / 000512159$
Teich/Bläker/Holtkamp-Endemann/ Jörgensen/Stallmach/Hohenberger 


\section{Statement of Ethics}

This study was conducted in compliance with the Independent Ethics Committee (IEC), informed consent regulations, and local regulatory requirements. The observational plan, informed consent documents, and any other appropriate study-related documents were approved by the applicable regional IEC of the Bavarian State Medical Board (Bayrische Landesärztekammer). All study documents were reviewed, approved, and/or permitted by the Paul-Ehrlich-Institut, Langen, Germany, before the study was initiated. Informed consent was obtained from all individual participants prior to study enrollment.

\section{Conflict of Interest Statement}

N.T. received personal fees from MSD during the study conduct and personal fees from Falk Foundation, Ferring Arzneimittel GmbH, Janssen, AbbVie, and Takeda. M.B., F.H.E., and E.J. received personal fees from MSD during the conduct of the study.
A.S. was a consultant for AbbVie, Amgen, Astellas, Falk Foundation, Ferring, MSD, and Takeda and had served as a paid speaker and/or scientific advisor on medical education events supported by MSD. S.H. is an employee of MSD.

\section{Funding Sources}

The PRODUCTIVE study was funded by MSD SHARP \& DOHME GmbH, Haar, Germany.

\section{Author Contributions}

N.T., M.B., F.H.E., E.J., and A.S. contributed to the generation, collection, assembly, analysis, and/or interpretation of the data. N.T. and S.H. drafted and revised the manuscript. All authors approved the final version of the manuscript. All authors had full access to all the data in the study and had the final responsibility for the decision to submit for publication.

\section{References}

1 Kalla R, Ventham NT, Satsangi J, Arnott ID Crohn's disease. BMJ. 2014 Nov;349:g6670.

2 Burisch J, Jess T, Martinato M, Lakatos PL. The burden of inflammatory bowel disease in Europe. J Crohns Colitis. 2013 May;7(4):32237.

3 Parra RS, Chebli JMF, Amarante HMBS, Flores C, Parente JML, Ramos O, et al. Quality of life, work productivity impairment and healthcare resources in inflammatory bowel diseases in Brazil. World J Gastroenterol. 2019 Oct;25(38):5862-82.

4 Bernstein CN, Loftus EV Jr, Ng SC, Lakatos PL, Moum B. Hospitalisations and surgery in Crohn's disease. Gut. 2012 Apr;61(4):622-9.

5 Sands BE, Anderson FH, Bernstein CN, Chey WY, Feagan BG, Fedorak RN, et al. Infliximab maintenance therapy for fistulizing Crohn's disease. N Engl J Med. 2004 Feb;350(9):87685.

6 Travassos WJ, Cheifetz AS. Infliximab: use in inflammatory bowel disease. Curr Treat Options Gastroenterol. 2005 Jun;8(3):187-96.

7 Danese S, Vuitton L, Peyrin-Biroulet L. Biologic agents for IBD: practical insights. Nat Rev Gastroenterol Hepatol. 2015 Sep;12(9): 537-45.

8 Vogelaar L, Spijker AV, van der Woude CJ. The impact of biologics on health-related quality of life in patients with inflammatory bowel disease. Clin Exp Gastroenterol. 2009; 2:101-9.

9 van der Valk ME, Mangen MJ, Leenders M, Dijkstra G, van Bodegraven AA, Fidder $\mathrm{HH}$, et al. Healthcare costs of inflammatory bowel disease have shifted from hospitalisation and surgery towards anti-TNFalpha therapy: results from the COIN study. Gut. 2014 Jan; 63(1):72-9
10 van der Valk ME, Mangen MJ, Severs M, van der Have M, Dijkstra G, van Bodegraven AA, et al. Evolution of costs of inflammatory bowel disease over two years of follow-up. PLoS One. 2016 Apr;11(4):e0142481.

11 Lo B, Vind I, Vester-Andersen MK, Bendtsen F, Burisch J. Direct and indirect costs of inflammatory bowel disease: ten years of follow-up in a Danish population-based inception cohort. J Crohns Colitis. 2020 Jan;14(1): 53-63.

12 Cohen RD, Yu AP, Wu EQ, Xie J, Mulani PM, Chao J. Systematic review: the costs of ulcerative colitis in Western countries. Aliment Pharmacol Ther. 2010 Apr;31(7):693-707.

13 Wolters FL, Russel MG, Sijbrandij J, Schouten LJ, Odes S, Riis L, et al. Disease outcome of inflammatory bowel disease patients: general outline of a Europe-wide population-based 10 -year clinical follow-up study. Scand J Gastroenterol Suppl. 2006(243):46-54.

14 Shen H, Lipka S, Katz S. Increased hospitalizations in elderly with inflammatory bowel disease on anti-tumor necrosis factor therapy but not increased infections: a community practice experience. J Crohns Colitis. 2014 Aug;8(8):898-9.

15 Xu J, Lin H, Feng X, Tang M, Shen J, Ran Z. Different therapeutic approaches on quality of life in patients with inflammatory bowel disease. BMC Gastroenterol. 2014 Nov;14: 199.

16 European Medicines Agency [Internet], Summary of product characteristics 2020, [cited 2020 March 19]. Available from: https:// www.ema.europa.eu/en/documents/product-information/remicade-epar-product-information_en.pdf.
17 Reilly MC, Gerlier L, Brabant Y, Brown M. Validity, reliability, and responsiveness of the work productivity and activity impairment questionnaire in Crohn's disease. Clin Ther. 2008 Feb;30(2):393-404.

18 Lichtenstein GR, Yan S, Bala M, Blank M, Sands BE. Infliximab maintenance treatment reduces hospitalizations, surgeries, and procedures in fistulizing Crohn's disease. Gastroenterology. 2005 Apr;128(4):862-9.

19 Leombruno JP, Nguyen GC, Grootendorst P, Juurlink D, Einarson T. Hospitalization and surgical rates in patients with Crohn's disease treated with infliximab: a matched analysis. Pharmacoepidemiol Drug Saf. 2011 Aug; 20(8):838-48.

20 Kane SV, Chao J, Mulani PM. Adherence to infliximab maintenance therapy and health care utilization and costs by Crohn's disease patients. Adv Ther. 2009 Oct;26(10):93646.

21 Feagan BG, Panaccione R, Sandborn WJ, D'Haens GR, Schreiber S, Rutgeerts PJ, et al. Effects of adalimumab therapy on incidence of hospitalization and surgery in Crohn's disease: results from the CHARM study. Gastroenterology. 2008 Nov;135(5):1493-9.

22 Loomes DE, Teshima C, Jacobs P, Fedorak $\mathrm{RN}$. Health care resource use and costs in Crohn's disease before and after infliximab therapy. Can J Gastroenterol. 2011 Sep;25(9): 497-502.

23 Costa J, Magro F, Caldeira D, Alarcão J, Sousa R, Vaz-Carneiro A. Infliximab reduces hospitalizations and surgery interventions in patients with inflammatory bowel disease: a systematic review and meta-analysis. Inflamm Bowel Dis. 2013 Sep;19(10):2098-110. 
24 Mao EJ, Hazlewood GS, Kaplan GG, PeyrinBiroulet L, Ananthakrishnan AN. Systematic review with meta-analysis: comparative efficacy of immunosuppressants and biologics for reducing hospitalisation and surgery in Crohn's disease and ulcerative colitis. Aliment Pharmacol Ther. 2017 Jan;45(1):3-13.

25 Lichtenstein GR, Yan S, Bala M, Hanauer S. Remission in patients with Crohn's disease is associated with improvement in employment and quality of life and a decrease in hospitalizations and surgeries. Am J Gastroenterol. 2004 Jan;99(1):91-6.

26 Reilly MC, Bracco A, Ricci JF, Santoro J, Stevens $T$. The validity and accuracy of the Work Productivity and Activity Impairment questionnaire: irritable bowel syndrome version (WPAI:IBS). Aliment Pharmacol Ther. 2004 Aug;20(4):459-67.

27 Wahlqvist P, Carlsson J, Stålhammar NO, Wiklund I. Validity of a work productivity and activity impairment questionnaire for patients with symptoms of gastro-esophageal reflux disease (WPAI-GERD): results from a cross-sectional study. Value Health. 2002 Apr;5(2):106-13.
28 Hoivik ML, Moum B, Solberg IC, Henriksen M, Cvancarova M, Bernklev T. Work disability in inflammatory bowel disease patients 10 years after disease onset: results from the IBSEN Study. Gut. 2013 Mar;62(3):368-75.

29 Steenholdt C, Brynskov J, Thomsen OØ, Munck LK, Christensen LA, Pedersen G, et al. Implications of infliximab treatment failure and influence of personalized treatment on patient-reported health-related quality of life and productivity outcomes in Crohn's disease. J Crohns Colitis. 2015 Nov;9(11):103242.

30 Loftus EV, Reinisch W, Panaccione R, Berg S, Alperovich G, Bereswill M, et al. Adalimumab effectiveness up to six years in adalimumabnaive patients with Crohn's disease: results of the PYRAMID registry. Inflamm Bowel Dis. 2019 Aug;25(9):1522-31.

31 Stark R, König HH, Leidl R. Costs of inflammatory bowel disease in Germany. Pharmacoeconomics. 2006;24(8):797-814.

32 van der Valk ME, Mangen MJ, Leenders $M$, Dijkstra G, van Bodegraven AA, Fidder HH, et al. Risk factors of work disability in patients with inflammatory bowel disease: a Dutch nationwide web-based survey: work disability in inflammatory bowel disease. J Crohns Colitis. 2014 Jul;8(7):590-7.
33 Juan J, Estiarte R, Colomé E, Artés M, Jiménez FJ, Alonso J. Burden of illness of Crohn's disease in Spain. Dig Liver Dis. 2003 Dec;35(12): 853-61.

34 Bernklev T, Jahnsen J, Henriksen M, Lygren I, Aadland E, Sauar J, et al. Relationship between sick leave, unemployment, disability, and health-related quality of life in patients with inflammatory bowel disease. Inflamm Bowel Dis. 2006 May;12(5):402-12.

35 De Boer AG, Bennebroek Evertsz' F, Stokkers PC, Bockting CL, Sanderman R, Hommes DW, et al. Employment status, difficulties at work and quality of life in inflammatory bowel disease patients. Eur J Gastroenterol Hepatol. 2016 Oct;28(10):1130-6.

36 Jacobs P, Bissonnette R, Guenther LC. Socioeconomic burden of immune-mediated inflammatory diseases: focusing on work productivity and disability. J Rheumatol Suppl. 2011 Nov;88:55-61.

37 Panes J, Lindsay JO, Teich N, Lindgren S, Colombel JF, Cornillie F, et al. Five-year safety data from OPUS, a European observational safety registry for adults with ulcerative colitis treated with originator infliximab [Remicade $^{\circledast}$ ] or conventional therapy. J Crohns Colitis. 2019 Sep;13(9):1148-57. 